Der folgende Artikel, den wir im Rahmen einer Medienpartnerschaft zwischen dem Schweizerischen Ärzteverlag EMH und den Organisatoren des 7. Schweizerischen eHealthcare Kongresses in Nottwil publizieren, wurde der SÄZ von den
Kongressverantwortlichen in der vorliegenden Form zugestellt. Die Redaktion nimmt bei Beiträgen, die Bestandteil einer Medienpartnerschaft sind, keinen Einfluss auf Themenwahl und Autor(en).

Die Redaktion

\title{
10 Jahre PKI im Gesundheitswesen
}

\author{
Das Schweizer Gesundheitswesen verfügt über eine eigene Public Key Infra- \\ structure (PKI). 80 Prozent der freipraktizierenden Ärztinnen und Ärzte sowie \\ 110 Spitäler, Labors und andere Institutionen des Gesundheitswesens nutzen \\ sie regelmässig.
}

Reinhard Dietrich

Korrespondenz:

Dr. sc. tech. Reinhard Dietrich

HIN Health Info Net AG

Technology Management

Pflanzschulstrasse 3

CH-8400 Winterthur

Tel. 0522351935

reinhard.dietrich@hin.ch
«Ich schicke Ihnen den Austrittsbericht noch heute mit der Post zu.» - «Per Post? Sie können ihn mir gerne auch aufs Mail legen.» - «Das geht leider nicht. Ich besitze nur ein normales E-Mail-Konto. Da dieses sensible Patientendaten nicht genügend schützt, darf ich es leider nicht benützen.» - «Ah, und ich dachte, dass heute jeder Arzt über ein verschlüsselungsfähiges Mail verfügt.»

So oder ähnlich verlaufen nach wie vor einzelne Telefongespräche. Ärzte versenden aus Datenschutzgründen sensible Patientendaten immer noch per Post oder allenfalls per Fax. Ganz so veraltet sind die Health Professionals in diesem Bereich jedoch nicht. Seit gut 10 Jahren verfügt das Schweizer Gesundheitswesen über ein gesichertes Netzwerk. Rund 80\% oder 11000 der freipraktizierenden Ärzte benützen die Schweizer E-Health-Plattform HIN Health Info Net regelmässig. Ebenfalls angeschlossen sind die Mitarbeiterinnen und Mitarbeiter von 110 Spitälern, Labors und weiteren Institutionen des Gesundheitswesens. Sensible Daten wie Patientendossiers, Röntgenbilder, Austrittsberichte oder E-Rezepte werden so sicher, datenschutzkonform und schnell über das Internet ausgetauscht.

\section{Hohe Useability \\ dank cleverer Clientsoftware}

Beim Aufbau der E-Health-Plattform wurden nebst den üblichen und notwendigen Massnahmen zur Sicherstellung des Datenschutzes und der Datensicherheit auch grosse Investitionen in die Nutzbarkeit (Useability) getätigt. Als Benutzer nehmen wir hohe Sicherheitsvorkehrungen oft als Hürde wahr, die es irgendwie zu überwinden gilt. Deshalb hat HIN nach
Lösungen gesucht, die die üblicherweise mit einer PKI verbundenen Useabilityprobleme vereinfachten. Durch den Einsatz einer cleveren Clientsoftware, die die Verwaltung der Zertifikate, die Integration in Applikationen, wie Browser, E-Mail-Client usw., für den Benutzer transparent übernimmt, konnten sowohl eine sehr hohe Datensicherheit als auch eine hohe Benutzerfreundlichkeit erreicht werden. Dies führte dazu, dass sich die Benutzer der HINPlattform wenig bewusst sind, dass im Hintergrund eine ausgewachsene PKI im Einsatz steht, die sich bezüglich Schlüssellänge und X.509Zertifikaten beispielsweise an die Vorgaben des ETSI (Technical Committee Electronic Signatures and Infrastructures) hält.

\section{Die PKI-Technologie steht im Hintergrund}

Der eigentliche Gebrauch der PKI-Lösung ist dadurch fast schon banal geworden. Der User wird beim Aufstarten des Computers lediglich einmal um ein Passwort gebeten. Die Clientsoftware wird gestartet und operiert danach nur noch im Hintergrund. Der Benutzer muss beim Versenden eines Mails nach wie vor lediglich 〈Senden〉 drücken. Der Client übernimmt ab da und kontrolliert die Empfängeradresse auf PKI-Zugehörigkeit. Bei PKI-Mails führt er die komplexen Arbeiten automatisch aus (Anklopfen bei der PKI, eindeutige Identifikation, SSL-Tunnelaufbau, Bezug öffentlicher Schlüssel, Verschlüsselung, nochmaliger SSL-Tunnelaufbau, Mailversand usw.). Einfacher geht es fast nicht. E-Mails, die an nichtgesicherte Domains geschickt werden sollen, sind möglich, verlassen den Computer aber unverschlüsselt. 


\section{Geschützte Internetapplikationen und Single Sign-on}

Gleichzeitig verfügt die E-Health-Plattform des Schweizer Gesundheitswesens auch über die Möglichkeit, webtaugliche Applikationen (KIS, RIS, LIS, PACS usw.) mittels Access Control Service zu sichern. Der Benutzer muss sich beispielsweise für den Zugriff auf die Seiten seines TrustCenters oder der Apotheke Zur Rose nur beim ersten Mal anmelden. Hat die Gegenseite seine Angaben verifiziert, erteilt er dem Benutzer ein Zugriffsrecht. Von da an kann der Benutzer mittels Mausklicks jederzeit auf die gesicherten Internetseiten. Die Authentifikation übernimmt wiederum die PKI-Technologie im Hintergrund.

\section{Zusammenarbeit mit regionalen E-Health-Projekten}

Die stark benutzer- und prozessorientierte Arbeitsweise hat wesentlich zur heutigen Akzeptanz und zum Wachstum der E-Health-Plattform HIN beigetragen. Von der grossen Verbreitung in der Ärzteschaft möchten mittlerweile auch die regionalen E-Health-Projekte profitieren. Daraus resultierte eine vertiefte Zusammenarbeit mit Rete Sanitaria im Tessin, den Regionen St. Gallen, Luzern, Genf und dem Fürstentum Liechtenstein. Sie alle nutzen Dienstleistungen der HIN-PKI, um die Vernetzung im Gesundheitswesen pragmatisch und kostengünstig umsetzen zu können.

\section{HIN - von Ärzten gegründet}

Das Unternehmen HIN Health Info Net wurde 1996 von der Verbindung der Schweizer Ärztinnen und Ärzte FMH und der Ärztekasse gegründet. Die ursprüngliche Aufgabe von HIN war, das Internet in die Arztpraxen zu bringen und ein gesichertes und datenschutzkonformes E-Mail für die Schweizer Ärztinnen und Ärzte zu entwickeln.

Heute zählt HIN 11000 Abonnentinnen und Abonnenten zu ihrem Benutzerkreis. 110 Spitäler, Kliniken, Labors, TrustCenter, Standesorganisationen und Krankenversicherungen sind ebenfalls mit der E-HealthPlattform verbunden. Das Winterthurer Unternehmen ist auch mit einer Geschäftsstelle in Lausanne vertreten.

Mit dem Sicherheitsspezialisten Arpage AG (ASAS Technologie) und der BlueCare AG (Management Support \& Administration) bestehen enge Kooperationen.

7. Schweizerischer eHealthcare Kongress Konferenz \& Fachausstellung

Mittwoch/Donnerstag, 26./27. September 2007 GZI Seminar- und Kongresshotel, Nottwil LU

Das Konferenzprogramm und weitere Informationen finden sich unter www.ehealthcare.ch. 